\title{
Il petrarchismo «sfumato» di Ernst Lubitsch
}

\author{
Fabrizio Denunzio
}

Università di Salerno

\section{Abstract}

Basato sull'analisi dei film Madame Du Barry (id., 1919) e Design for Living (Partita a quattro, 1933), e su alcuni lavori teorici di Ernst Lubitsch, il saggio dimostra la relazione esistente tra l'opera del regista e il Canzoniere di Petrarca.

In particolare, attraverso il concetto di «sfumatura», il lavoro evidenzia il trattamento al quale Lubitsch sottopone due dei fondamentali elementi della poesia petrarchesca: la catastrofe e la donna. La "sfumatura", idea in parte elaborata dal filosofo Walter Benjamin nei Passagenwerk, in Lubitsch agisce sia al livello della narrazione sia al livello visivo del montaggio.

Una volta divenute immagini in movimento, le idee di Petrarca, grazie alla «sfumatura» lubitschiana, perdono la loro «durezza» testuale e liberano il loro potenziale politico e «spettacolare».

Così la catastrofe, che nel Canzoniere si riferisce all'evento della Peste nera, nei film di Lubitsch è connessa agli eventi politici della Rivoluzione russa e dell'ascesa del nazismo. La donna, che nelle poesie del Petrarca è sempre l'immagine fantasma di Laura, in Lubitsch acquisisce un sofisticato erotismo.

Parole chiave: montaggio, sfumatura, catastrofe, erotismo, eventi storici.

\section{Abstract}

The paper shows the relationships between Ernst Lubitsch and Petrarca's Canzoniere, based on the analysis of two of his films (Madame Du Barry, 1919 and Design for Living, 1933) and of some of his essays. The concept of shade allows the director to manipulate some of the fundamental elements of Petrarca's poetry, such as the Catastrophe and the Woman. This idea of shade, already partially introduced by Walter Benjamin in Passagenwerk, is crucial both in Lubitsch's concept of narrative and in his visual editing style.

Once become motion pictures, Petrarca's themes lose their textual "hardness" and release their political and «spectacular» potential, thanks to Lubitsch «shading style». So that the Catastrophe, that in the Canzoniere refers to the Plague, in Lubitsch's interpretation is related to the event of the Russian Revolution and the ascent of Nazism. The Woman, that in Petrarca's poetry is nothing else than the phantom of Laura, in Lubitsch's works acquires a sophisticated eroticism.

Key words: editing, shade, catastrophe, woman, eroticism, historical events. 


\section{PARTE PRIMA}

\section{Walter Benjamin: iattura, rivoluzione e montaggio}

Agli inizi del 1930 Walter Benjamin scrive una lettera particolarmente significativa a Gerhard Scholem. Per rompere il silenzio in cui è caduto e riuscire così ad aggiornare l'amico di sempre sia sulla sua situazione attuale sia sui progetti futuri, si maschera dietro il francese. Protetto dalla maschera di una lingua straniera Benjamin trova il modo per dire a Scholem che ritarda ulteriormente il tanto auspicato viaggio in Palestina e che non gli è più possibile lo studio dell'ebraico.

Liquidate così le questioni nelle quali era direttamente coinvolto l'amico, Benjamin valuta gli ultimi due anni della sua attività. Riscontra di non avere raggiunto il fine prepostosi sin da quando gli era stata rifiutata la libera docenza dalla Facoltà di filosofia dell'Università di Francoforte, quello cioè di diventare «il primo critico della letteratura tedesca». ${ }^{1} \mathrm{E}$ riuscito sì a farsi una posizione nella critica, ma non è ancora diventato il primo dei suoi addetti.

Chiarito anche questo argomento Benjamin parla dei lavori futuri. L'editore Rowohlt si è dichiarato disponibile a pubblicare una sua raccolta di saggi, da qui l'impegno a realizzare due studi rispettivamente sullo stile liberty e sulla situazione e sulla teoria della critica.

Arrivati a questo punto la lettera prende un altro tono. Se fin qui Benjamin ha dato l'impressione di stilare un distaccato resoconto della sua vita all'amico più caro, ora, iniziando a parlare dei Passages una vibrazione comincia ad attraversare la sua voce: "[...] ciò che mi impegnerà è il mio libro Pariser Passagen [...] è il teatro di tutte le mie lotte e di tutte le mie idee». ${ }^{2}$ Questa incrinatura prodottasi inaspettatamente all'interno dell'economia generale di una lettera fin qui ai limiti della pura formalità nonostante la discreta confidenza che Benjamin avesse col destinatario, è ciò che ai nostri occhi la rende estremamente significativa.

Questa crepa testuale ci permette di mettere a fuoco e intensificare un dettaglio: nella lettera Benjamin pensa ai Passages come luogo «spettacolare» dei suoi combattimenti. Se ci è dato conoscere l'insieme di concetti che il filosofo tedesco convoca e fa recitare sulla scena della sua opera, un po' più difficile risulta immaginarci i termini dei conflitti da lui sostenuti allorquando questi ultimi non vengano banalmente interpretati come il complesso delle difficoltà materiali contro cui l'esistenza di Benjamin ha sempre guerreggiato.

Allora, contro cosa ha lottato Benjamin per tutta la vita di così ineluttabile da fare dei Passages, opera della vita (iniziata il 1927 e arrestatasi solo con la morte nel 1940), il luogo della sua rappresentazione drammatica?

Infanzia berlinese si conclude con un capitolo intitolato «L'omino con la gobba». Personaggio appartenente al modo delle favole, Benjamin dice di aver- 
lo incontrato sin da bambino e, per quanto non l'abbia mai visto, sa di essere caduto nella trama "maledetta» del suo sguardo. Fu la madre a svelare involontariamente a Benjamin il significato reale del "gobbetto»: "Malestro [ungeschickt] ti saluta". In tedesco l'aggettivo ungeschickt significa tanto maldestro quanto sfortunato. ${ }^{3} \mathrm{Da}$ allora, da quando è stato guardato per la prima volta dal "gobbetto", Benjamin non se ne è mai più distaccato e con lui, attraverso di lui, si è impiantata nella sua vita la malasorte: «Il gobbetto mi precedeva dappertutto. E, precedendomi, egli mi contrastava il cammino». ${ }^{4}$

Hannah Arendt ha tracciato con estrema precisione l'inevitabile puntualità con cui ogni episodio della vita di Benjamin si è venuto a trovare in perfetta concomitanza con «il culmine della malasorte». 5 A noi non resta che prenderne atto e tornare ai Passages.

Con questa chiave di lettura possiamo avanzare l'ipotesi che nella sua ultima grande opera il filosofo tedesco abbia voluto dare finalmente forma a questo combattimento inesausto portato avanti fin dalla più tenera età contro la iattura, così che tutti i Passages, alla fine, ne risultano intrisi.

Appurati i termini dello scontro sostenuto da Benjamin, ora bisogna capire la natura di questa malasorte. Questione oziosa e al limite del ridicolo: la malasorte è sempre e solo malasorte! Ma facciamo attenzione. La iattura che permea di sé i Passages non riguarda solamente l'opera, ma coinvolge direttamente il suo fruitore. Questo vuol dire che la malasorte dei Passages invischia il suo lettore e così facendo dimostra di essere una iattura salutare. Sì perché la sfortuna che promana dall'opera di Benjamin non ha niente a che fare con la superstizione popolare, essa non vuole fare altro che vincolare il suo fedele compulsatore ad un pensiero che sia rivoluzionario. Quindi, l'unica vera disgrazia che ci può capitare «sperimentando» i Passages nella nostra vita e nella nostra attività è quella di ritrovarci vincolati ad una rivoluzione.

\section{II}

Leggiamo nella cartella $N$. Elementi di teoria della conoscenza, teoria del progresso dei Passages: "[...] adottare nella storia il principio del montaggio».6 Per quanti sforzi si possano fare per ridurre ad un mero episodio il rapporto di Benjamin con il cinema, culminato nella stesura del magistrale saggio L'opera d'arte nell'era della sua riproducibilità tecnica, e riportare così la sua opera nelle acque quiete della filosofia, di fronte a questa annotazione cruciale dei Passages si dovrebbe iniziare a pensare che Benjamin, resosi conto dell'insufficienza concettuale della sua tradizione culturale, abbia chiesto al cinema non solo degli strumenti con cui pensare il suo presente, ma delle «armi» con cui distruggere proprio quella tradizione inservibile.

3. Su questo punto cfr. la nota del traduttore in Hanna ARENDT, Walter Benjamin: l'omino gobbo e il pescatore di perle, in ID., Il futuro alle spalle, Bologna: il Mulino, 1995, p. 100.

4. Walter Benjamin, Infanzia berlinese, Torino: Einaudi, 1973, p. 124.

5. Cfr. Hanna ARENDT, cit., p. 43-103.

6. Walter Benjamin, $I$ «passages» di Parigi, vol. I, Torino: Einaudi, 2002, p. 515. 
E qui sta il nucleo incandescente dei Passages, la sua iattura rivoluzionaria più profonda: per essere realmente moderni, per farla finita con la Tradizione del sapere, Benjamin non indica nessuna metodologia di sorta, sperimenta direttamente la distruzione introducendo nella teoria le moderne forme create dal linguaggio cinematografico. E dato che la genesi di tali forme dipende per intero dall'avvento delle masse sulla scena della Storia e dai loro bisogni culturali, possiamo individuare il senso di un gesto rivoluzionario nella capacità che esso ha di immettere la cultura di massa nel ceppo inaridito della Tradizione. Si capisce quanto una deflagrazione sia inevitabile. Quindi, dopo l'avvento del cinema non c'è Nome della Tradizione che possa rimanere fissato alla sua datità storica. Quello di Petrarca meno di tutti.

\section{III}

Benjamin dimostra di avere una padronanza effettiva della dimensione concettuale del montaggio quando, in un'annotazione di poco precedente a quella citata, riflettendo sugli adattamenti cinematografici del Faust goethiano, istituisce delle differenze: tra il poema del Faust e il film sul Faust c'è un abisso incolmabile (differenza di natura), eppure tra riduzioni filmiche sullo stesso soggetto esistono egualmente della disparità abissali (differenza di grado). Ciò che conta alla fine, aggiunge Benjamin, «[...] non sono mai i "grandi contrasti", ma solo quelli dialettici, che sembrano estremamente simili a delle sfumature». ${ }^{7}$

A essere messi in relazione in queste pagine dei Passages sono il montaggio e la dialettica. Si avrebbe facile gioco a voler riconoscere in queste affermazioni l'influenza di Hegel da un lato e di Sergej M. Ejzenstejn dall'altro, un riconoscimento tanto più semplice quanto più autorizzato dallo stesso autore: nella lettera commentata Benjamin dice che per arrivare a strutturare in maniera solida i Passages dovrà studiare attentamente «certi aspetti di Hegel»; ${ }^{8}$ ritornato dal suo viaggio a Mosca, Benjamin scrive sul numero dell' 11 Marzo del 1927 della «Literarische Welt» un violento saggio in difesa del cinema sovietico come risposta alla lettura di Bronenosec Potëmkin (La corazzata Potëmkin, 1926) di Sergej M. Ejsenstejn avanzata dal critico Oscar Schmitz in cui conferma di conoscere alla perfezione il film e l'autore. ${ }^{9}$

Quindi Hegel come filosofo supremo della dialettica, Ejzenstejn come regista e teorico del montaggio come "dialettica della forma cinematografica». ${ }^{10}$ Quanta ragione ci sarebbe nel riconoscere in questi nomi i referenti di Benjamin. Eppure un elemento ci invita a sragionare: la sfumatura. I contrasti dialettici come sfumature, il montaggio come sfumatura. Questa idea non appartiene né ad Hegel

7. Ibid, p. 513 (corsivo nostro).

8. Walter BENJAMIN, Lettere 1913-1940, cit., p. 178.

9. Cfr. Walter Benjamin, Replica a Oscar A. H. Schmitz, in ID., Opere complete II. Scritti 19231927, Torino: Einaudi, 2001, p. 617-621.

10. S. M. EJZENSTEjN, La forma cinematografica, Torino: Einaudi, 1986, p. 49. 
per il quale la dialettica, l'aufhebung, ha sempre significato la conservazione dell'essenziale di ciò che si sopprime ${ }^{11}$ (rimanendo così nella sostanza una operazione di morte), ${ }^{12}$ né ad Ejzenstejn per il quale il montaggio si deve risolvere in ultima istanza in una sintesi organica delle diverse fasi di cui è composto il film. ${ }^{13}$

Benjamin intuisce che il montaggio e la dialettica cinematografica possono ispirarsi ad un principio differente da quello che, fino ad allora, gli era stato assegnato da Hegel e dai registi sovietici: la sfumatura. L'intuizione da sola, però, non basta.

A questo punto interviene Ernst Lubitsch. In un testo elaborato nel 1947, ma pubblicato solo nel 1962 sul numero 25 della rivista Filmculture, il grande regista tedesco riflette a ritroso sulla sua opera e pensando soprattutto ai film in costume del periodo tedesco - Madame Du Barry (id., 1919), Sumurun (id., 1920), Anna Boleyn (Anna Bolena, 1920) — dice di averli pensati in funzione sottrattiva rispetto a quelli dello stesso tipo fatti "dalla scuola italiana allora in voga, e che faceva tanto "Gran-Opera". Io cercavo invece di togliere qualsiasi alone operistico [...] Il mio scopo era quello di raccontare la vicenda attraverso le sfumature». ${ }^{14}$

E Lubitsch a prendere la parola, è lui che formalizza quanto in Benjamin rimaneva purtroppo allo stadio dell'intuizione. Sfumare significa togliere. $\mathrm{Ma}$ se le cose stanno così abbiamo realmente fatto un progresso? Non siamo ancora nei pressi di Hegel visto che proprio l'aufheben hegeliano deriva dal latino tollere (togliere), ${ }^{15}$ e che proprio un altro grande teorico e regista sovietico, Vsevolod Pudovkin, della statura di Ejzenstejn, ha concepito il montaggio dialettico come lo strumento con cui togliere nel film tutto ciò che è superfluo alla visione dello spettatore? ${ }^{16}$

Per Lubitsch sottrarre non vorrà mai dire imporre il negativo della morte, quindi della tristezza, come avviene per la soppressione hegeliana, né tanto meno eliminare il superfluo dalla visione spettatoriale, tutt'altro.

Un film organizzato in base alla sfumatura non farà altro che sottrarre solidità alle cose, fino ad arrivare alla leggerezza, al divertimento, alla pura evasione da ogni consistenza: "[...] so bene che teatro e cinema sono basati su fondamenti di vanità». ${ }^{17} \mathrm{Un}$ film costruito attraverso il principio della sfumatura sarà un film fatto di indizi, di tenui tracce, di vaghe allusioni e di creative ellissi, e in questa fitta trama di seducente inconsistenza vedremo come l'immagine (fantasma) della donna (petrarchesca) vi giocherà un ruolo fondamentale.

11. Cfr. A. Kojève, La dialettica e l'idea della morte in Hegel, Torino: Einaudi, 1991, p. 50.

12. Su questo punto cfr. Gilles Deleuze, Nietzsche e la filosofia e altri testi, Torino: Einaudi, 2002 (in particolare si veda il capitolo V, "Il superuomo contro la dialettica», p. 221-288)

13. Cfr. S. M. Ejzenstejn, cit., p. 181.

14. Ernst LubitsCH, La mia carriera, in F. BONO (a cura di), Lubitsch, Roma: Officina Edizioni, 1992, p. 66 (corsivi nostri).

15. Cfr. B. Moroncini, «Tradurre è perdonare?», Studium, n. 5, 2003, p. 729.

16. Cfr. V. I. Pudovkin, "Il regista e il materiale cinematografico», in ID., La settima arte, Roma: Editori Riuniti, 1961, p. 34-40.

17. Ernst LuBITSCH, Gli attori che ho diretto in America, in F. BONO, cit., p. 61. 


\section{PARTE SECONDA}

\section{Il petrarchismo sfumato di Ernst Lubitsch}

Il petrarchismo di Lubitsch si può sintetizzare in un unico postulato concettuale: sfumare l'immobilismo ontologico del Petrarca.

Si capisce subito da questa apertura che la presenza del poeta all'interno del cinema lubitschiano per noi si può misurare soltanto in base alla sua evanescenza: se c'è un qualcosa come un petrarchismo cinematografico in Lubitsch esso è percepibile solamente in quei momenti in cui va in fumo; del resto, in questa sede, anche l'analisi dei film lubitschiani, a sua volta, deve essere svolta esclusivamente in base a queste sfumature. In tal senso parliamo di petrarchismo «sfumato».

Lubitsch adotta la stessa posizione politica del Petrarca nei confronti degli eventi catastrofici della storia: fare dell'immagine femminile l'elemento con cui trascrivere sul piano simbolico il senso della distruzione. ${ }^{18}$

Entrambi i film "petrarcheschi» che intendiamo analizzare nascono in circostanze di questo tipo: Madame Du Barry è proiettato all'UFA Palast am Zoo, la più grande sala di Berlino, la sera del 18 settembre 1919, a quasi un anno di distanza dall'inizio della rivoluzione socialista tedesca e pochi mesi dopo la nascita della Repubblica sovietica bavarese; ${ }^{19}$ Design for Living (Partita a quattro) esce a New York il 22 novembre del 1933, agli inizi dello stesso anno, esattamente il 28 febbraio, Hitler con la proclamazione del Decreto per la protezione del popolo e dello Stato aveva sospeso la validità costituzionale delle libertà personali garantite dalla Costituzione di Weimar ${ }^{20}$ stabilendo così la definitiva ascesa del totalitarismo nazista.

Come il Petrarca aveva di fronte a sé la Peste nera, ${ }^{21}$ così Lubitsch, per ben due volte nel corso della sua vita, viene posto dinanzi a delle fratture storiche epocali. Allo stesso modo in cui il Petrarca utilizzava l'immagine della donna per ricomporre il senso simbolico del disastro pestilenziale, così Lubitsch fa della donna la protagonista dei suoi film al fine di ricavare dalle sciagure sovversive un simbolo $^{22}$ femminile di comprensione degli accadimenti: nel caso di

18. Per vedere come tale meccanismo ricorra puntualmente nella «società di massa» spagnola e napoletana del '600 cfr. Juan Antonio Maravall, La cultura del Barocco. Analisi di una struttura storica, Bologna: il Mulino, 1985 e Giorgio Alfano, Per dolore ruinando, Introduzione di G. Alfano - M. BARBATO - A. MAZzUCCHI (a cura di), Tre catastrofi. Eruzioni, rivolta e peste nella poesia del Seicento napoletano, Napoli: Cronopio, 2000, p. 7-31.

19. Cfr. la testimonianza autobiografica degli eventi raccontata da Ernst Toller nel suo Una giovinezza in Germania, Torino: Einaudi, 1972, p. 118-174. (Ringrazio Vittorio Dini per avermi suggerito la lettura del testo).

20. Cfr. Giorgio Agamben, Stato d'eccezione, Torino: Bollati Boringhieri, 2003, p. 24-25.

21. Cfr. Ugo DotTI, Vita di Petrarca, Roma-Bari: Laterza, 1992.

22. Sulla simbolicità del cinema cfr. Gino FrEZZA, «Dal volto al monolito. Fra simbolo e cinema», in A. CICALESE - A. LANDI (a cura di), Simboli, linguaggi e contesti. Quaderni del Dipar- 
Madame Du Barry e della catastrofe rivoluzionaria comunista si tratta della sartina Jeanne che da semplice modista diventa la preferita di Luigi XV; nel caso di Design for Living e della calamità nazista, abbiamo il complesso mondo sentimentale di Gilda, una giovane agente pubblicitaria innamorata di due uomini.

Partito dalla stessa posizione del Petrarca, Lubitsch deve necessariamente farla sfumare, consapevole di servire il cinema e non la poesia.

Dato il difficile codice comunicativo con cui è articolata l'opera petrarchesca, l'universalismo a cui aspira — visto che il poeta deve rivolgersi universalmente a tutti perché solo così può sperare di adempiere la sua missione di unificatore dell'identità culturale di un paese lacerato come l'Italia del suo tempo- non può essere che potenziale, e dal momento che un vero e proprio livello democratico di massificazione la poesia del Petrarca non l'ha mai raggiunto, la sua universalità, rimanendo «in potenza», si è immobilizzata.

Pur ponendo l'immagine della donna sullo stesso livello culturale di elaborazione simbolica del Petrarca, Lubitsch non può correre il rischio di immobilizzarne l'universale comprensione. Da qui l'esigenza di sfumare l'immobilismo petrarchesco. Ma come? Sicuramente non basta dire che in questo compito Lubitsch è agevolato dalla tecnologia di riproduzione automatica delle immagini alla quale si deve il movimentismo della visione cinematografica. Non è perché dispone di immagini-movimento che il grande regista tedesco riesce a sfumare l'immobilismo della pagina scritta petrarchesca e a raggiungere così l'universalismo. Tutto dipende a servizio di quale idea di cinema le immagini-movimento sono poste.

Sulla rivista Lichtbild-Bühne del 16 ottobre 1920, Lubitsch scrive una lettera polemica alla grande attrice danese Asta Nielsen, da lui diretta in Rausch (1919), adattamento cinematografico di un dramma di August Strindberg. Questa lettera è un "testo" molto importante perché raramente il regista tedesco scrive del suo lavoro e, soprattutto, si lascia andare a polemiche con gli attori dei suoi film.

Evidentemente nelle accuse rivoltegli da Asta Nielsen Lubitsch legge qualcosa di più di un semplice attacco personale alla sua trasposizione della pièce strindberghiana. Nel rimprovero di non aver rispettato la poesia del drammaturgo danese, Lubitsch sente che ad essere chiamata in causa non è semplicemente la relazione conflittuale tra due forme espressive diverse, ma la propria posizione teorica all'interno del cinema, e su questo punto il regista ha le idee ben chiare: "L'arte di Strindberg è intellettuale [...] I problemi intellettuali non si potranno mai portare sullo schermo!». ${ }^{23} \mathrm{O}$ meglio, visto che Lubitsch non si è mai esentato dal pensare le catastrofi del nostro secolo (oltre a quelle prese qui in considerazione, si pensi al modo in cui affronta la disastrosa condizione del reduce dalla Prima guerra mondiale in The Man I Kil-

timento di Scienze della Comunicazione (2/2002), Università di Salerno, Roma: Carocci, 2002, p. 87-147.

23. Ernst Lubitsch, Una lettera ad Asta Nielsen, in F. Bono, cit., p. 55. 
led, L'uomo che ho ucciso, 1932), si potranno affrontare problematiche «intellettuali» solo a patto che la soglia evasiva del divertimento non precipiti mai nella paralizzante astrazione dell'intellettualismo. Dovendo scrivere nel 1930 un articolo per The World Film Encyclopedia sui vari aspetti della direzione cinematografica Lubitsch approfondisce ulteriormente questa idea: «[...] è necessario avere sempre nuove idee, trovare nuovi modi per mantenere desto l'interesse del pubblico». ${ }^{24}$

Quindi, quella con cui abbiamo a che fare nell'opera di Lubitsch è una vera e propria fondazione cinematografica dell'evasione, idea rivoluzionaria per l'intero sistema delle comunicazioni poiché può prendere consistenza solo se arriva ad implicare nelle sue strutture espressive una soggettività spettatoriale. Per tale motivo Lubitsch fa del pubblico non "l' in più della creazione», ma "l'insieme», la parte fondamentale dei suoi film dal momento che permette "agli spettatori di costruire essi stessi la sceneggiatura, assieme a lui, mentre il film scorre sullo schermo». ${ }^{25}$

Molto è stato detto sull'emigrazione dei registi austriaci e tedeschi dalla vecchia Europa verso il nuovo continente americano: se per la seconda ondata di fuoriusciti — quella di Fritz Lang — la salita al potere dei nazisti negli anni ' 30 può offrire una valida motivazione all'esilio, più difficile risulta individuare le cause che hanno presieduto alla partenza del primo flusso migratorio, quello a cui prese parte Lubitsch. Semplici vantaggi economici offerti da Hollywood? Miraggio di un successo ancora più grande di quello conseguito in Europa? Solo in parte. Diciamo piuttosto che nell'America a cavallo tra gli anni Venti e gli anni Trenta Lubitsch trova una condizione socio-culturale nettamente diversa da quella europea, ${ }^{26}$ tale da fargli funzionare in modo decisamente migliore la sua idea di evasione cinematografica.

In questo periodo storico gli Stati Uniti riescono a realizzare una corrispondenza impeccabile tra i bisogni di una massa di consumatori estetici profondamente motivati a sfruttare al massimo tutte le potenzialità fantasmagoriche del proprio tempo libero e un'industria seriamente intenzionata a sfruttare questa esigenza. ${ }^{27}$ Ciò vuol dire che sul territorio americano, negli anni presi in considerazione, la fabbrica coincide con la società, ossia, la produzione industriale di sogni è realmente sottomessa ai desideri degli spettatori, meglio ancora, rispetto al Capitale la soggettività spettatoriale non si trova per nulla soggetta a meccanismi di dominio ma, al contrario, può affermare, rivendicare e vedere realizzate le sue più profonde esigenze antropologiche.

Se nel suo lavoro all'UFA (la più grande casa di produzione cinematografica della Germania) Lubitsch doveva in qualche modo suscitare una soggettività spettatoriale affinché potesse implicarla nelle sue strategie evasive (da qui il ricorso alla spettacolarità dei drammi storici), in America non ha

\section{Ernst LUBITSCH, Il lavoro del regista, in F. BONO, cit., p. 60.}

25. François Truffaut, Lubitsch, in ID., I film della mia vita, Venezia: Marsilio, 1998, p. 67.

26. Cfr. A. AbruZZESE, Forme estetiche e società di massa, Venezia: Marsilio, 1992, p. 187-237.

27. Ibid, p. 189. 
più bisogno di questo espediente perché l'implicabile è già pienamente esplicato: un pubblico «evasivo» disponibile ad ogni avventura. Ė degno di nota che ad Hollywood Lubitsch non abbia mai girato un film dalle enormi risorse finanziarie.

Così, paradossalmente, mentre il regista praticava l'evasione cinematografica in una splendente solitudine europea fu visto come reazionario agli occhi del più attento critico e teorico di sinistra del cinema tedesco del periodo di Weimar. E non a caso le invettive di Siegfried Kracauer si concentrarono proprio su Madame Du Barry, il cui incanto deve non poco al fasto delle scenografie (gli interni della corte di Versailles), alla magnificenza degli ambienti (i giardini in cui gioca Luigi XV), al lusso dei costumi (in particolare quello delle cortigiane), alla spettacolare orchestrazione delle masse (sia di quelle rivoluzionarie dei giacobini, sia quelle dei nobili danzatori).

Così nello stesso tempo in cui Lubitsch era impegnato nella strutturazione dell'evasione filmica in un'opera come Madame Du Barry, con la quale cercava di fare dell'immagine femminile lo «stilo» e la "superficie» con cui e su cui trascrivere in termini simbolico-universali il disastro relativo alla rivoluzione tedesca del 1919 (nello spostamento sociale intrapreso da Jeanne in realtà si metaforizza il suo spostamento dal piano disastrato della storia a quello intellegibile dell'allegoria), Kracauer reagiva alla fondazione lubitschiana invocando le leggi economiche, le cause ideali, i processi storici ${ }^{28}$ per connotare l'estraneità della pellicola a quel buon senso comune a cui ogni film "politico» dovrebbe attenersi.

Allora, a differenza di quanto avveniva con l'universalismo potenziale dell'immagine femminile del Petrarca, se quella di Lubitsch riesce ad essere «in atto" universalmente intellegibile e ad offrirsi come luogo di elaborazione simbolica delle distruzioni a cui è chiamata a rispondere, questo dipende dal fatto che il regista ha sempre accordato una natura eminentemente evasiva alla macchina cinematografica.

Lì dove per universalismo non si intende nulla di diverso dal movimento con cui lo spettatore si singolarizza, si traccia un proprio percorso di comprensione all'interno della rappresentazione collettiva messa in essere dal cinema. ${ }^{29}$ E lì dove per evasione si intende proprio il movimento assicurato dalla capacità che hanno le immagini-movimento di produrre godimento spettatoriale, di incrinare magicamente l'anima e farla fuori uscire da sé.

Non sempre il cinema è riuscito a raggiungere questa edenica condizione e quando lo ha fatto, non è mai riuscito ad andare oltre l'orizzonte definito da Lubitsch.

28. Cfr. S. Kracauer, Da Caligari a Hitler. Una storia psicologica del cinema tedesco, Torino: Lindau, 2001, p. 96-102.

29. Cfr. Jacques DERRIDA, "Il cinema e i suoi fantasmi», in aut aut, 309, 2002, p. 58. 


\section{II}

Se il concetto di evasione permette a Lubitsch di sfumare l'immobilità ontologica dell'universalismo potenziale del Petrarca, la forma visiva con cui il regista riesce a sfumare l'immobilismo figurativo dell'immagine della donna petrarchesca è il fuori-campo.

Se nel primo caso era il permanere nella potenza causato dalla difficoltà annessa al codice comunicativo poetico ad immobilizzare l'intelligibilità universale della poesia petrarchesca, nel secondo è la sbarratura della sessualità a produrre la rigidezza dell'immagine femminile del Petrarca.

Anche in questo caso si capirebbe ben poco dell'immagine fantasma della donna lubitschiana facendo appello alla natura fantômale dell'immagine cinematografica. Come la natura meccanica del movimento da sola non arriva a spiegare il tipo di movimento teorico che presiede all'idea di cinema di Lubitsch finché non se ne individua il concetto di evasione, così la semplice natura spettrale dell'immagine cinematografica non è sufficiente per definire la fantasmaticità della donna creata dal regista fino a quando non si risale alla sua concezione della sessualità e alla sua maniera formale di trattarla.

La fantasmatizzazione petrarchesca è uno sbarramento della sessualità.

Con questo non vogliamo dire che dal Canzoniere sia bandita la dimensione sessuale anzi, di suo desiderio e di suo piacere il poeta ne fa trasudare in abbondanza ad ogni canzone, ma è l'immagine di Laura, nonostante i profondi turbamenti che riesce a produrre nel Petrarca, a non essere sufficientemente sessualizzata proprio perché definitivamente fantasmatizzata: «Ci è nella bellezza corporale un certo non so che, visibile ma intangibile, che sta nel corpo e appare come un di là del corpo, senza contorni né determinazioni, di una natura così eterea e vaporosa che ci dà una prossima immagine dell'anima [...] il corpo ti si spoglia innanzi di ogni parte terrena e greve, divenuto spirituale, voglio dire simile ad un fantasma, ad un'ombra». ${ }^{30}$

Se questa Laura fredda, quieta, priva di vita, non «contentava» il pur romantico De Sanctis, figuriamoci se poteva soddisfare quel sottile e finissimo amante della tumultuosa corporeità femminile che è Lubitsch.

L'operazione formale del regista tedesco consiste nello sfumare l'immobilismo sessuale dell'immagine fantasma di Laura non tanto andando a risessualizzare l'immagine della donna dandole un corpo dotato di tutti gli attributi della seduzione, quanto fantasmatizzando nell'assenza del fuori-campo la presenza della sessualità femminile.

Design for Living è un'opera magistrale. Non solo perché la forma del fuoricampo raggiunge la sua massima definizione conseguendo la sfumatura dell'immobilismo tipico dell'immagine fantasma petrarchesca, ma anche perché l'intero film, come avveniva in precedenza con Madame Du Barry, è posto al servizio dell'elaborazione simbolica del disastro storico rappresentato dall'ascesa nazista. 
Un giudizio dello psicanalista Sergio Finzi aiuta ad inquadrare meglio questo aspetto fondamentale di Design for Living. Forse perché estremamente entusiasta del film Finzi giunge a dire: «Mi viene a volte da pensare che la coincidenza dell'avvento del nazismo e dell'uscita nello stesso anno 1933 di un film come Partita a quattro di Lubitsch, un film che presenta le vicissitudini di un sodalizio erotico e intellettuale che viola i confini della coppia senza pagare il prezzo [...] della redenzione cristiana, non sia da considerarsi accidentale e che il nazismo sia stato la risposta potente come il maglio mondiale dell'ortodossia sessuale a quella fragile, delicata operetta con i suoi due tulipani in un vasettom. ${ }^{31}$

Piuttosto è vero il contrario, visto che il film esce diversi mesi dopo la salita al potere dei nazisti. Ma ciò che importa in questi giudizi ai limiti del delirio dettati dal puro piacere spettatoriale, è che si riesce a trovare in essi più saggezza e senso critico di quanto si creda e di quanto sia lecito aspettarsi di trovarne in chi si occupa a titolo istituzionale di cinema.

Si sbaglierebbe moltissimo a pensare che Lubitsch affronti esplicitamente il problema nazista solo in To Be or Not To Be (Vogliamo vivere!, 1942, film sulle vicissitudini di una compagnia teatrale di attori ebrei durante l'occupazione tedesca della Polonia) e nel relativo articolo comparso sul «New York Times» del 29 marzo del $1942 .{ }^{32}$ In questa stupenda e geniale pellicola, piuttosto, il regista ritorna sull'argomento e in modo non meno radicale di quanto faccia in Design for Living, come del resto, dopo Madame Du Barry, ritornerà radicalmente sugli esiti implosivi della rivoluzione comunista sovietica in Ninotchka (id., 1939, è la storia di un commissario politico donna della Russia stalinista che si «corrompe» venendo a contatto con il consumismo dell'Occidente capitalista).

Se negli anni Trenta il nazismo edifica la sua dimensione ideologica su valori soldateschi e militari, ${ }^{33}$ non potendo fare altro di conseguenza che promuovere una dimensione rigidamente ortodossa della sessualità - la perversione sadica dei nazisti è una necessità tutta interna al cinema italiano, si pensi a Portiere di notte (1974, di Liliana Cavani) e a Salò o le 120 giornate di Sodoma (1975, di Pier Paolo Pasolini $)^{34}$ —, dal suo canto Lubitsch elabora questo trau-

31. S. FINZI, La scienza dei vincoli. Opus reticulatum: reti e vincoli in psicoanalisi, Bergamo: Moretti \& Vitali, 2000, p. 81.

32. Cfr. Ernst LuBiTSCH, To Be or Not To Be, in F. Bono, cit., p. 64.

33. Cfr. Georges Bataille, Nietzsche e il nazionalsocialismo, in ID., Su Nietzsche, Milano: ES, 1994, p. 199.

34. Su questo punto fanno chiarezza Primo LeVI «Film e svastiche» in ID., Opere I, Torino: Einaudi, 1997, p. 1218 («Quanto alle SS, per lo più non erano dei mostri, né dei mandrilli idioti, né dei bellimbusti pervertiti: erano dei funzionari dello Stato", corsivo nostro), e la decisiva intervista di Michel Foucault, Sade, sergeant du sexe, in ID., Dits et Écrits (1954-1988), tome II (1970-1975), Paris: Éditions Gallimard, 1994, p. 818-822. Riferendosi ai film della Cavani e di Pasolini Foucault dice: «C'est une erreur historique totale. Le nazisme n’a pas été inventé par les grands fous érotiques $\mathrm{du} \mathrm{XX}$ siècle, mais par les petits-bourgeois les plus sinistres, ennuyeux, dégoûtants qu'on puisse imaginer. Himmler était vaguement agronome, et il avait épousé une infermière. Il faut comprendre que les camps de concentration sont nés de l'imagination conjointe d'une infirmière d'hôpital et d'un éleveur de poulets. Hôpital plus basse-cour: voilà le fantasme qu'il y avait derrière les camps de concentration [...] 
Éros absent [...] Le problème qui se pose est de savoir pourquoi aujourd'hui nous nous imaginons avoir accès à certains fantasmes érotiques à travers le nazisme». È su questo aspetto del suo film che Pasolini non sembra essersi interrogato a sufficienza. Con non poche ragioni si potrebbe dire che Salò o le 120 giornate di Sodoma una volta riportato alla clausura culturale italiana nella quale e contro la quale matura, sfugge al giudizio foucaultiano. In effetti è proprio lo stesso Pasolini nelle diverse interviste rilasciate sul film a tirare in ballo il cambiamento intervenuto nei costumi sessuali della società italiana, ossia, il fatto che nel 1975 , a differenza di quanto avvenisse per la Trilogia della Vita (Il Decameron, 1971; I Racconti di Canterbury, 1973; Il fiore delle mille e una notte, 1974), la gioiosa visione del sesso come "compenso [...] alla repressione» (Pier Paolo PASOLINI, Per il cinema, tomo II, Milano: Mondadori, 2001, p. 2064) doveva essere abbandonata a causa dell'atteggiamento conciliativo che si iniziava ad accordare al problema sessuale: «La tolleranza [...] avrebbe reso il sesso triste e ossessivo", ibid. Inoltre, la coprofagia di Salò era da intendersi, dopo l'aumento del livello consumistico registrato in Italia negli anni ' 70 , come la violenza con cui «i produttori costringono i consumatori a mangiare merda» (ibid, p. 3021). È in queste stesse interviste che Pasolini, però, mentre lega il film al contesto situazionale italiano, lascia intendere che la sessualità proposta dal potere nazista si leghi al modello sessuale perverso elaborato da Sade: "[... tutto il sesso che c'è in Salò (e ce n'è in quantità enorme) è anche la metafora del rapporto del potere con coloro che gli sono sottoposti [...] la riduzione del corpo a cosa» (ibid, p. 2065); «E un potere che manipola i corpi in modo orribile e che non ha nulla da invidiare alla manipolazione fatta da Hitler: li manipola trasformando la coscienza, cioè nel modo peggiore; isituendo dei nuovi valori alienanti» (ibid, p. 3027); «[...] il potere è codificatore e rituale, e anche i gesti erotici lo sono, e siccome appunto la gesticolazione è sempre la stessa, e si ripete eternamente uguale, risulta che la gestualità sodomitica è la più tipica [...] quella che meglio riassume la ripetitività dell'atto", ibid. Foucault, anche a volerne ridimensionare la forza del giudizio, non è del tutto lontano dall'aver focalizzato il problema, in fondo non leggere con la sufficiente attenzione Sade porta Pasolini a non avere una precisa conoscenza del nazismo. Anche per questo Levi non esitava a pronunciare un durissimo giudizio su Salò: «[...] mi è dispiaciuto molto come film, mi è sembrato un rigurgito, l'opera di un uomo disperato, infatti Pasolini era disperato. E io non amo la disperazione. Mi pare che la disperazione paralizzi. Credo che sia un film che ha nuociuto, questo [...] Non era così, non è vero che fosse così. Questa ferocia totale non è esistita» (Primo Levi, Conversazioni e interviste, Torino: Einaudi, 1997, p. 251). Forse è proprio per salvaguardare uno dei nostri più originali intellettuali comunisti moderni dalla constatazione di questo «erreur totale» (dare un erotismo eretico al nazismo quando in realtà questo non supera la più povera sessualità immaginata da una piccola borghesia provinciale) che matura in tutta la sua evidenza non a caso in una opera cinematografica, che i curatori italiani dell'Archivio Foucault hanno ben pensato di escludere dalla raccolta Sade, sergeant du sexe quando, proprio in base ai criteri con cui hanno organizzato la selezione, aveva il pieno diritto di rientrarvi: non rappresenta forse questa intervista (forma espressiva privilegiata dai curatori) una tappa evolutiva fondamentale della riflessione di Foucault su Sade tanto che — sempre che si voglia tenere presente l'insieme complessivo degli interventi teorici del filosofo sul libertino francese così da registrarne gli scarti più sensibili-, ce ne offre una «nuova formulazione» (esigenza principale dei curatori è quella di seguire le diverse fasi dello sviluppo di Foucault anche su di uno stesso tema)? Non è forse in questa intervista che per la prima volta Foucault — dopo avercene disegnato il ritratto di teorico della sragione (Storia della follia), di "semiologo" estremo del linguaggio e di quello della sessualità in particolare (Prefazione alla trasgressione e Il linguaggio all'infinito in Scritti letterari), di ultimo esponente della rappresentazione discorsiva classica (Le parole e le cose), di figura mostruosa in cui si incarna l'autodistruzione della natura (Gli anormali) — definisce Sade come il formulatore di un «érotisme propre à une société disciplinaire», ma, soprattutto indica la necessità di uscire dal sadismo per creare finalmente una nuova forma erotica non disciplinare? E non chiede Foucault al cinema di concorrere attivamente a questa opera di creazione con l'invenzione di nuove immagini piuttosto che con la rappresentazione degli effetti «alienanti» del potere? 
ma storico formalizzando l'immagine di una donna centrata su di una forte eterodossia sessuale (una relazione a tre non era assolutamente usuale a quei tempi), ai margini dell'eresia così da decostruire in tempo reale quegli stessi valori che si diffondono dal disastro nazista. Ricordiamo che Goebbels in un incontro con gli operatori del cinema tenutosi a Berlino il 13 marzo del 1933, subito dopo essere stato nominato Ministro della Propaganda nazista, raccomanda ai cineasti il rispetto di «alcune regole» ${ }^{35}$ e, nei discorsi successivi, dichiara che i film tedeschi si devono attenere sempre ai principi di un «sano» realismo così da mostrare ambienti e uomini "quali si trovano nella realtà». ${ }^{36}$

Forse è proprio per questo che in Design for Living si ride così tanto: Lubitsch deve aver capito che alla pesante ferraglia di una sessualità da "caserma» regolativa e basata su di un realismo "ortopedico", si poteva solamente rispondere con la leggerezza degli effimeri riverberi di un piacere corporeo discretamente eccessivo.

$\mathrm{E}$ a questa posizione Lubitsch non rinuncerà neanche in seguito quando il confronto con il nazismo si farà diretto ed esplicito come in To Be or Not To $B e$. Anche in questo caso il regista preferirà la linea evasiva del divertimento a quella «impegnata» della denuncia, eppure, con quanta intelligenza decostruirà ulteriormente il totalitarismo nazista evitando sempre di connotarlo sessualmente: «Non ho mostrato camere di tortura, né primi piani di nazisti sovreccitati, che brandiscono una frusta e roteano gli occhi concupiscenti. I miei nazisti sono diversi; è molto tempo che hanno superato questo stadio». ${ }^{37}$ Lezione inascoltata!

Ora, come costruisce formalmente, quindi in modo visivo Lubitsch la sessualità eterodossa della protagonista di Design for Living, così da sfumare l'immobilità dell'immagine femminile del Petrarca?

«Niente sesso. Ricordatevi che è un impegno d'onore!». Più di un rimedio cautelativo a cui Gilda (interpretata dalla bravissima e affascinante Miriam Hopkins) sembra fare ricorso per salvare almeno l'amicizia che la lega ai suoi due amanti (George/Gary Cooper e Tom/Fredric March), viste le dinamiche possessive scatenatesi in loro dopo la prima tessitura sessuale della relazione a tre (una volta capito che la ragazza è andata a letto con entrambi, ognuno cerca di farne la propria donna iniziando ad odiare l'altro), in questa semplice affermazione della ragazza si sostanzia il procedimento lubitschiano (estremamente ironico) di visualizzazione della sessualità: niente sesso... che sia visibile nel campo dell'inquadratura filmica.

Questo non vuol dire che la sessualità occupa interamente lo spazio del fuori-campo, se così fosse si verrebbe a creare un puerile manicheismo visivo che porterebbe ad un'opposizione morale tra la pudica assenza del sesso in campo e la sua libertaria e trasgressiva presenza nel fuori-campo.

35. J. Goebbels, cit. in S. SoccI, Fritz Lang, Roma: Il Castoro Cinema, 1995, p. 55-56.

36. J. Goebbels, cit. in L. EISNER, Lo schermo demoniaco. Le influenze di Max Reinhardt e dell'espressionismo, Roma: Editori Riuniti, 1983, p. 225.

37. Ernst LubitsCH, To Be or Not To Be, cit., p. 64. 
Naturalmente tutto ciò non succede in Lubitsch. Il sofisticato regista tedesco non può che scegliersi la via più difficile: in Design for Living la sessualità femminile costituisce l'immagine fantasma che lega i due differenti regimi della visione appartenenti rispettivamente al campo e al fuori-campo, in questo senso Gilda dice di volere "tutti e due» gli uomini che la corteggiano intendendo che la sua immagine femminile ha bisogno della comunicazione costante tra i due livelli visivi per realizzarsi.

Il raccontare attraverso le sfumature, di cui parlavamo nell'apertura del nostro lavoro, significa proprio questo: Lubitsch fa in modo che al racconto della relazione sessuale manchi sempre qualcosa, che essa non sia mai raccontata del tutto ${ }^{38}$ che ogni volta in essa appaia un indizio ${ }^{39}$ di un vuoto, a questo punto è il fuori-campo la zona segreta che si fa carico di raccontare quella mancanza, quel niente, quel non finito che Lubitsch ha così sapientemente fatto fiorire nel cuore della sua storia.

Basta un abito da sera indossato al mattino presto, ad esempio. Tom rientra a Parigi subito dopo aver ricevuto la notizia del sopravvenuto fidanzamento tra Gilda e George. Ferito in cuor suo dal doppio tradimento degli amici che a sua insaputa, senza coinvolgerlo in tempo sulla fatale decisione, hanno deciso di rompere il triangolo, si reca a fargli visita. Arrivato nell'appartamento in cui la coppia vive, scopre che George è via per lavoro, la sua attività di pittore sta andando molto bene, ora fa il ritrattista per la "grassa» borghesia americana stanziata sul territorio francese. Trova, invece, Gilda: i due non impiegano molto tempo a capire che la passione è come al solito travolgente. Non atteso, il mattino seguente George si presenta a casa: l'entusiasmo si spegne di colpo quando, accortosi dell'inadeguatezza dell'abbigliamento di Tom — «Abito da sera per colazione? Cos'è l'ultima moda londinese?»— deduce che i due hanno fatto l'amore. Il capo di vestiario è l'indizio che, richiamando il fuori-campo "sessuale» della notte precedente, apre il vuoto nella certezza che George aveva nella fedeltà di Gilda.

Ancora. Esausta del comportamento litigioso dei suoi partner, incapaci di viversi il rapporto a tre senza gelosie e competizioni, avvelenando di conseguenza la sua innocente, leggera e gioiosa serenità corporea, Gilda decide di sposarsi con l'improbabile signor Max Plunkett, interpretato magistralmente dal grandissimo Edward Everett Horton. La prima notte di nozze Max ha fretta di "consumare» il matrimonio, animato in questo e dalla bellezza di Gilda e dalla consapevolezza della sua libertà sessuale. Arrivati a casa dopo la celebrazione del rito trovano l'abitazione piena di omaggi floreali, tra gli altri, un adorabile e minuscolo vaso con due fiori, dalla lettura del biglietto sappiamo che George e Tom dalla lontana Cina si sono ricordati dell'avvenimento. Gilda si innervosisce, inizia a pensare ai due, prende a calci il vasetto, nel frattempo Max trova il modo di ricondurla ai suoi doveri coniugali, la porta nella stanza da letto. Forse perché dispiaciuta del gesto violento avuto 
nei confronti del regalo degli amici, forse perché estremamente vicina al pericolo di trascorrere una notte di sesso con Max, Gilda esce dalla camera nuziale è ricompone i fiori. Questo atto riconciliativo molto probabilmente le permette di prendere coraggio e di rientrare in stanza. La nottata trascorre, ad uscire dalla camera questa volta è Max, come è lui questa volta a prendersela coi fiori di Tom e George, dal che si deduce la delusione del signor Plunkett sugli auspicati esiti sessuali della prima notte di nozze con Gilda. In questa sequenza il raccordo tra campo e fuori-campo, tra la visibilità dell'anticamera e l'invisibilità della stanza da letto, è giocato dall'indizio dei fiori: l'accanimento con cui Max li scalcia nel campo visibile dell'anticamera connota il fallimento del suo agognato rapporto sessuale avvenuto nello spazio invisibile del fuori campo della camera da letto le cui porte, come sempre in Lubitsch, sono rigorosamente serrate.

Pensiamo, infine, alla sequenza esemplare in cui Gilda e George fanno ritorno a casa dopo aver salutato Tom in partenza per Londra dove sarà rappresentata una sua commedia, e rimangono soli. Girano nervosamente per la stanza, cercano soluzioni possibili per scongiurare di andare a letto assieme, ma niente: già sdraiata Gilda, mentre George si tiene in fuori-campo, invoca le cause di forza maggiore in virtù delle quali anche gli impegni più onerosi come quelli presi da loro si possono derogare. Lubitsch fa seguire a questa resa delle armi una più che eloquente dissolvenza su nero.

E così facendo costruisce l'immagine fantasma della donna: fantasma perché come la sua sessualità non è visibile nella presenza del campo dal momento che manca ogni contatto dei corpi, così, data la sostanziale invisibilità del fuori-campo, essa rimane egualmente invisibile nell'assenza del fuori-campo, più del nero della dissolvenza non si riesce a vedere. Eppure, nel rinvio continuo che in tal modo si istituisce tra le due zone dell'(in)visibile cinematografico, la sessualità femminile è sensibilmente presente. Se Gilles Deleuze definisce l'insieme di procedimenti inferenziali che si producono da questo uso fantasmatico della femminilità lubitschiana «immagine-ragionamento», ${ }^{40}$ lo fa perché non dimentica mai che essi sono volutamente introdotti nell'immagine al fine di attivare $\mathrm{i}$ «ragionamenti» dell'unica soggettività che potrà mai effettuarli ed, effettuandoli, completarli di significato: quella spettatoriale.

Quindi, pur rimanendo ancorato al livello del fantasma, Lubitsch riesce a sfumare l'immobilismo sessuale in cui il Petrarca ha irrigidito l'immagine di Laura.

Sarà un caso che nell'ultimo film della sua vita (The Lady in Ermine, La signora in Ermellino, 1948, finito da Otto Preminger a causa della morte di Lubitsch) il regista faccia innamorare il protagonista dell'immagine pittorica di una nobil donna del passato il cui fantasma ha la strana abitudine di animarsi e lasciare il quadro per correre in aiuto dei suoi discendenti in pericolo?

La grande operazione visivo-concettuale compiuta da Lubitsch sul Petrarca è solo uno dei tanti motivi che ci fanno ammirare e amare la sua opera. 